\title{
Biodiversité et végétation pastorale
}

\author{
Ph. Daget ${ }^{1}$ J. Poissonet ${ }^{1}$
}

\section{Mots-clés}

Biodiversité - Flore - Pâturage - Zone tropicale - Mesure - Végétation Méthode - Mauritanie - Burkina Faso Cameroun - Tchad.

\section{Résumé}

Un rappel des normes de qualification de la richesse spécifique de la flore des pâturages est fait, illustré d'exemples mauritaniens, burkinabés, camerounais et tchadiens. Les rapports entre la richesse floristique et la productivité, les potentialités du milieu, la pression humaine sont envisagés et un modèle global de variabilité est proposé.

\section{INTRO DUCTION}

Une des «mesures » (4) les plus fréquentes de la biodiversité d'un peuplement végétal est la richesse floristique, parfois même considérée comme la diversité ; en fait, il s'agit d'une mesure de la diversité parmi un ensemble d'autres $(1,23)$ pour lesquelles il a été montré qu'elles pouvaient toutes être exprimées par une seule expression générale $(3,14) \mathrm{N} \alpha$, fonction d'un paramètre unique $\alpha$.

\section{RICHESSE FLO RISTIQUE ET DIVERSITE}

\section{Richesse stationnelle}

La richesse floristique stationnelle correspond au nombre de diversité d'ordre zéro. Elle est souvent donnée comme une caractéristique de l'état de la flore. Un jugement de valeur sur la flore des pâturages est souvent établi d'après le nombre d'espèces présentes sur l'aire minimale et son extension, donc dans l'aire stationnelle ; il doit l'être par référence à l'échelle suivante (8) :

- flore très pauvre, lorsqu'il y a moins de 10 espèces ;

- flore pauvre, lorsqu'il y a de 11 à 20 espèces ;

- flore moyenne, lorsqu'il y a de 21 à 30 espèces ;

- flore assez riche, lorsqu'il y a de 31 à 40 espèces ;

- flore riche, lorsqu'il y a de 41 à 50 espèces ;

- flore très riche, lorsqu'il y a plus de 51 espèces.

On peut ajouter «flore raréfiée » avec moins de 5 espèces et «particulièrement riche » avec plus de 70 espèces.

1. CIRAD-EMVT/CNRS, Campus international de Baillarguet, BP 5035, 34032 Montpellier Cedex 1, France

\section{Quelques exemples}

- Steppes de Mauritanie centrale

Ce premier exemple est emprunté à une exploration de Monod (18) en Mauritanie centrale. Trente-neuf stations de steppe très ouverte (ou Badiah marginal) ont été analysées entre le 20 décembre et le 20 janvier 1974. Parmi ces stations, il y en avait 16 où la flore était raréfiée, 16 où elle était très pauvre et 7 où elle était pauvre (tableau I : a).

\section{- Bahr el Ghazal du Tchad}

Les observations sont celles de Yosko (25) ; il s'agit de steppes boisées au Nord Est du lac Tchad, analysées en 1993. Le tableau I (b) donne les résultats obtenus à partir des 39 premières stations de son échantillonnage. Parmi ces stations, il y en avait 2 où la flore était raréfiée, 11 où elle était très pauvre, 23 où elle était moyenne et 3 où elle était assez riche.

- Steppes du Nord Cameroun

Le tableau I (c) donne les résultats obtenus à partir de 39 des stations analysées par Gaston (12) en 1981. Parmi elles, il y en avait 19 où la flore était raréfiée, 19 où elle était très pauvre et une seule où elle était moyenne.

- Savanes de l'Adamaoua au Cameroun

Les résultats de 39 des stations analysées par Yonkeu (24) en 1993 dans l'Adamaoua vers $1400 \mathrm{~m}$ d'altitude sont donnés dans le tableau I (d). Ici, il y avait 3 flores moyennes, 8 assez riches, 8 riches, 10 très riches et 10 particulièrement riches.

\section{Variabilité locale}

Lorsque plusieurs stations ont été analysées par des relevés standards dans la même région, ou dans le même degré-carré, il est intéressant d'examiner la variabilité interstationnelle de la biodiversité. Cela permet, en particulier, de déterminer si telle ou telle biodiversité mesurée (4) dans telle ou telle station est représentative de la situation régionale ou s'il s'agit de cas particuliers. 


\section{Tableau I}

Caractéristiques de la richesse spécifique dans quatre groupes de 39 stations agrostologiques

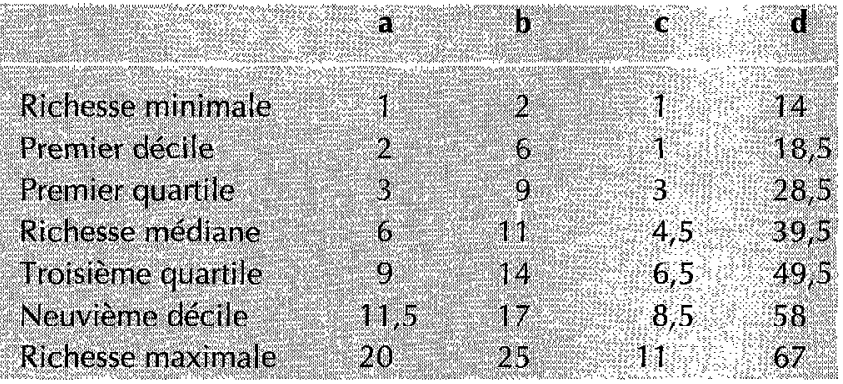

La liste des chiffres correspondant aux mesures faites peut être caractérisée par les méthodes de la statistique descriptive ; en particulier par la suite des fractiles usuels :

- le minimum et le maximum,

- la médiane (valeur qui divise les données en deux lots égaux),

- le premier décile (10 p. 100 des données lui sont inférieures) et le neuvième (10 p. 100 lui sont supérieures),

- le premier quartile ( 25 p. 100 des données lui sont inférieures) et le troisième ( 25 p. 100 lui sont supérieures).

La confrontation, même seulement visuelle, de ces diverses valeurs apporte d'intéressants renseignements sur la variabilité locale de la biodiversité et, lorsque plusieurs séries sont disponibles, sur la variabilité inter-rćgionalc. Cc type de confrontation est souvent suffisant, mais il peut être complété par une représentation graphique (graphe de Box et Wistler ou Boxplot (19)) ou par une analyse de variance selon le modèle de Kruskall-Wallis.

\section{VARIATION DE LA RICHESSE FLORISTIQUE ET CONDITIONS STATIONNELLES}

\section{Diversité et productivité}

Il a été écrit que le nombre d'espèces, donc la biodiversité, est un marqueur de la productivité des formations pâturées.

Mais c'est une notation trop rapide. En effet, si elle peut être considérée comme vraie au niveau régional (figure 1), il n'en va pas de même au niveau stationnel. C'est ainsi que, si les sebkhas sursalées avec une seule ou parfois deux ou trois espèces, peuvent être considérées comme une des végétations africaines les moins productives, les bourgoutières, avec la même biodiversité, sont parmi les plus productives (7). Par ailleurs, le moment où les observations sont faites a une importance primordiale, comme le montre la comparaison des colonnes a et $\mathrm{c}$ du tableau précédent ; une confrontation sans précaution indiquerait que les steppes du nord du Cameroun sont plus pauvres et plus arides que les badiah de moyenne Mauritanie ! En fait, il faut préciser que les observations de Gaston ont été faites en fin de saison sèche, de sorte qu'il ne subsistait guère que les espèces ligneuses...

Par ailleurs, dans les végétations étudiées par Yonkeu (24) au Cameroun, les plus riches, dont la biodiversité est la plus élevée, sont celles des falaises. Enfin, les cultures les plus intensives présentent une biodiversité très faible résultant d'une éradication systématique des espèces autres que la plante cultivée productrice de rendement agricole.

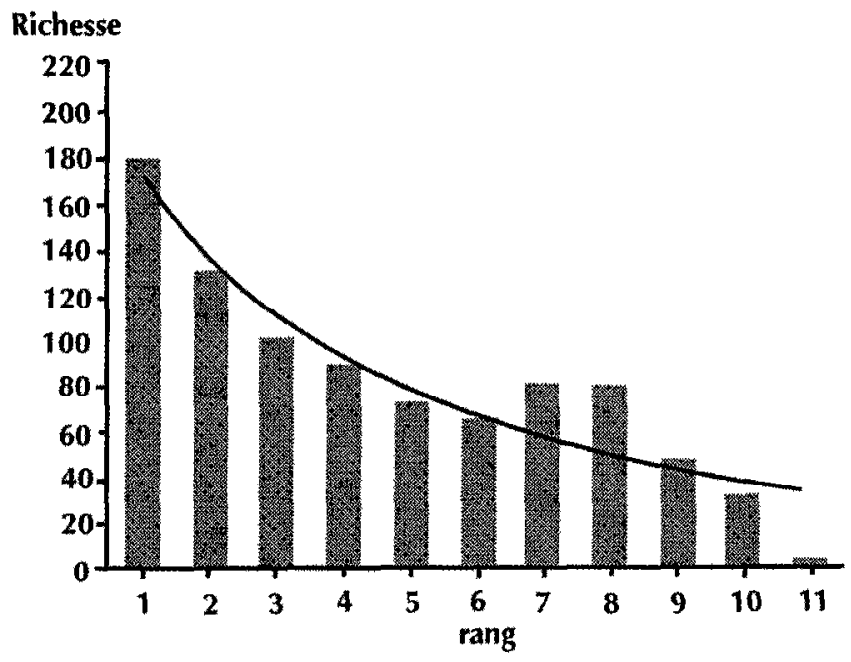

Figure 1 : relation entre la latitude et la richesse spécifique sur la Côte mauritanienne.

Le rang est la latitude de demi-degré en demi-degré à partir de l'embouchure du Sénégal (d'après Dia A.T., CIRAD-EMVT, Montpellier, France, 1996).

\section{Diversité et stabilité}

McNaughton a écrit « There is something almost mythological about the hypothesis that greater species diversity in ecological communities is associated with greater community stability » (17), malgré les travaux fondamentaux de May (16). Il faut prendre en compte tout l'écosystème et sa dynamique. Ainsi, la faible diversité d'une culture intensive est instable et cède la place à la forte diversité de la forêt secondaire en passant par les stades intermédiaires de la jachère. Le processus n'est pas simple et uniforme ; il présente une succession de maximums et de minimums relativement complexe (7). La diversité tout aussi faible des végétations hygrophiles qui entourent les mares correspond, quant à elle, à des végétations très stables.

\section{Diversité et potentialités du milieu}

La richesse spécifique est souvent considérée comme déterminée par les conditions de milieu (20). En particulier, on dit souvent que plus un sol a de potentialités, donc plus il est fertile et bien alimenté en eau, et plus sa richesse floristique est élevée. C'est ainsi que la biodiversité augmente avec l'épaisseur de placage sableux recouvrant les glacis pierreux du nord du Burkina Faso (tableau II).

\section{Tableau II}

Richesse spécifique des pâturages du Burkina Faso et état de la surface du sol (d'après Sanon et coll., IN.ERA, Ouagadoudou, Burkina Faso, 1994)

Pas de placage et glacis plerteux
Pas de placage et glacis gravillonnaire
Pas de placage et glacis throno sableux
Glacis avec un placage sableux peu épais
Glacis avec un placage sableux noyen
Glacis avec un placage sableux mportant


Il est clair que la relation existant entre la biodiversité et la production est plus complexe. Dans les cultures, plus la production est élevée et plus la biodiversité est faible. Cela résulte de la lutte contre la concurrence effectuée par le cultivateur lorsqu'il éradique les mauvaises herbes ; même dans les végétations naturelles cette relation est souvent en défaut. C'est ainsi que la végétation la plus productive de l'Afrique sahélienne, les bourgoutières à Echinochloa stagnina (11), est aussi celle dont la flore est la plus pauvre : moins de trois espèces, souvent une seule.

\section{Diversité et pression humaine}

On parle souvent des liaisons entre la biodiversité et l'action humaine (22) pour déplorer une action dépressive, mais des zooécologues (2) ont montré qu'une activité humaine modérée était créatrice d'une biodiversité maximale, correspondant à un paysage en mosaïque (10) dont le grain est moyen, les taches nombreuses et de formes variées et la structure verticale étagée. La biodiversité diminue quand l'artificialisation (13) augmente, car les structures horizontales et verticales du paysage s'estompent progressivement. Elle diminue aussi quand l'artificialisation baisse, donc quand le terroir est abandonné ; en effet, dans ces conditions le paysage se ferme en se simplifiant (20 ; Dulieu comm. pers.). Ainsi, dans l'échelle de variation de ce paramètre écologique qu'est l'artificialisation du milieu (ou intensité de la pression humaine et, puisqu'il s'agit ici de pâturages, de la charge), la biodiversité commence par augmenter, passe par un maximum pour une pression modérée, puis diminue jusqu'à des valeurs faibles.

\section{REPRESENTATION SCHEMATIQ U E DE LA VARIABILITE D'EN SEM BLE}

Il en est ainsi quel que soit le paramètre écologique ; la biodiversité diminue quand augmente :

- la sécheresse stationnelle,

- l'humidité stationnelle,

- la teneur du sol en sel,

- la durée de la saison sèche,

- la rigueur de la saison froide,

- etc.

Une représentation théorique schématique de cette variation est donnée par une courbe « en cloche » pluri-factorielle (figure 2).

Figure 2 : modèle de variation de la richesse spécifique avec les paramètres mésologiques.

$X$ : humidité stationnelle ; $Z$ : sécheresse ; $Y$ : pression humaine ; W : profondeur du sol, etc.
Toutefois, ce «modèle » ne se prête pas aisément à une modélisation opérationnelle. En effet, en ce qui concerne l'humidité stationnelle, par exemple, la diminution de la richesse floristique, liée à l'augmentation de l'influence de l'eau, telle qu'elle ressort des relevés de FLOTROP (5), met en évidence l'importance de l'existence d'une couche d'eau affleurante, mais aussi de sa pérennité au cours de l'année, de son épaisseur et de sa variation spatiale, de l'existence ou non d'une zone d'eau libre, de la nature des accès à la mare, tant par le bétail que par les animaux sauvages (et bien entendu de leur espèce), de l'histoire de l'appropriation des rives et de la surface aquatique (existence d'une mise en valeur par des rizières, abandon de ces dernières et modalités de cet abandon, éventuellement existence de techniques de restauration). Et le fonctionnement des autres axes est aussi complexe..

Dans les études agrostologiques locales, les paramètres majeurs peuvent être dégagés par une analyse factorielle des correspondances, ou par une " analyse écologique » (6). Une représentation plus simple, appuyée sur ces seuls paramètres, est alors possible. Ainsi, Sanon et coll. (21) ont-ils mis en évidence que la biodiversité agrostologique diminue dans quatre directions majeures :

- diminution de la profondeur et augmentation de la mobilité du revêtement sableux ;

- augmentation de l'importance des cailloux et des cuirasses ;

- augmentation de l'humidité stationnelle globale ;

- augmentation de la pression humaine.

La figure 3 montre l'organisation de ce jeu d'influences sur la richesse floristique stationnelle.

\section{CONCLUSION}

La prise en compte de la biodiversité est devenue, depuis quelques années, un des poncifs de l'approche écologique de l'aménagement et sa préservation un des points majeurs des recommandations des grands bailleurs de fonds. Mais, au terme de cette analyse, deux conclusions s'imposent.

Figure 3 : organisation d'ensemble de la variation de la biodiversité agrostologique (richesse floristique stationnelle) dans le Territoire de Menegou au Burkina Faso.

Redessiné d'après Sanon et coll., IN.ERA, O uagadoudou, Burkina Faso, 1994. 
En premier lieu, même réduit à son squelette, c'est-à-dire à sa richesse spécifique, le concept de biodiversité n'est pas aussi simple qu'il n'y paraît pour ceux dont l'écologie n'est pas la spécialité.

En second lieu, le fonctionnement de la réalité biologique dont il rend compte peut être très complexe, et ce sont les gradients de biodiversité qu'il convient de prendre en compte et d'interprèter selon les paramètres écologiques majeurs ; une valeur isolée n'aurait guère de sens.

Comprendre la biodiversité d'un site, d'une région, implique une prise en compte approfondie, non seulement de l'ensemble des paramètres mésologiques, mais aussi de leur appréhension par les populations humaines utilisatrices de ces végétations, donc de l'ensemble des paramètres socio-culturels et économiques. C'est toute la difficulté, mais aussi la grandeur, de l'écologie appliquée.

\section{BIBLIO GRAPH IE}

1. BLONDEL J., 1979. Biogéographie et écologie. Paris, France, Masson, $172 \mathrm{p}$.

2. BLONDEL J., 1995. Biogéographie - Approche écologique et évolutive. Paris, France, Masson, 297 p.

3. DAGET Ph., 1980. Le nombre de diversité de Hill, un concept unificateur dans la théorie de la diversité écologique. Acta 0 ecol., 0 ecol. Gener., $1: 51-70$.

4. DAGET Ph., 1982. Sur le concept de mesure et son application en écologie générale. Vie et Milieu, $32: 61-68$.

5. DAGET Ph., 1995. "FLOTROP", une base de données agro-pastorales sur l'Afrique tropicale au CIRAD-EMVT. Revue Élev. M éd. vét. Pays trop., $48: 281-282$.

6. DAGET, Ph., GODRON M., 1988. Analyse fréquentielle de l'écologie des espèces dans les communautés. Paris, France, Masson, 172 p.

7. DAGET Ph., GUELLY T., 1996. La biodiversité dans la reconquête forestière post-cultural. In : Dynamique à long terme des écosystèmes forestiers intertropicaux. Bondy, France, ORSTO M, p. 301-304.

8. DAGET Ph., POISSONET J., 1991. Prairies et pâturages, méthodes d'études. Montpellier, France, Institut de Botanique, $354 \mathrm{p}$.

9. DIA A.T., 1996. Végétation du littoral mauritanien. Montpellier France, CIRAD-EMVT, 74 p. (Rapport CIRAD-EM VT n $96-019$ )

\section{Summary}

D aget Ph., Poissonet J. Biodiversity and pastoral vegetation

This review serves as a reminder of qualification standards for specific richness of the pastoral flora, illustrated with Mauritanian, Burkinabian, Chadian and Cameroonian examples. Relations between the floristic richness and productivity, potentiality of the environment, human pressure are considered and a global model of variability is proposed.

Key words: Biodiversity - Flora - Grazing - Tropical zone Measurement - Vegetation - M ethod - Mauritania - Burkina Faso - Cameroon - Chad.
10. FORMAN R., GODRON M., 1986. Landscape ecology. New York, USA, Wiley, $619 \mathrm{p}$.

11. FRAN COIS J., RIVAS A., COMPERE R., 1989. Le pâturage semiaquatique à Echinochloa stagnina (Retz.) P. Beauv. Bull. Rech. agron. Gembloux, 24 : 145- 189.

12. GASTON A., 1981. La végétation du Tchad - Évolutions récentes sous des influences climatiques et humaines. Thèse doct., Univ. Paris XII, France, $333 \mathrm{p}$.

13. GODRON M. ed., 1968. Code écologique pour le relevé de la végétation et des milieux. Paris, France, CNRS.

14. HILL M., 1973. Diversity and evenness: a unifying notation ans its consequences. Ecology, 45 : 427-432.

15. KLEIN D., YONKEU S., 1984. Rapport annuel 1983-84 du CRZ de Wakwa, N'Gaoundéré, Cameroun.

16. MAY R., 1975. Patterns of species abundance and diversity. In: Cody M., Diamond J. eds., Ecology and evolution of communities. Cambridge, Mass., U SA, Belknap Press, p. 81- 120.

17. MCNAUGHTON S., 1988. Diversity and stability. Nature, 333 (6170): 204-205.

18. MONOD Th., 1974. Spectre de modes de dissémination dans I'Adrar mauritanien (Sahara occidental). Candollea, 29 : 401-425.

19. READ S., READ T., 1988. Statistical issues in setting product speciations, XI - Boxplots. HP Journal, 39: 11.

20. RIPPSTEIN G., 1986. Etude sur la végétation de I'Adamaoua. Maisons-Alfort, France, IEM VT, 380 p. (Etudes et synthèses de I'IEMVT $\left.\mathrm{n}^{\circ} 14\right)$

21. SANON P., KONE N., LIEHOUN E., POISSO NET J., MORANT Ph., SOMDA M., 1994. Etude agrostologique du terroir de Menegou. 0 uagadougou, Burkina Faso, Inst. Etudes Rech. agric., 80 p.

22. VAN DER MAAREL E., 1973. Plant species diversity in relation to management. CR 11th Symp. British ecol. Soc.: 45-63.

23. VEIRA DA SILVA J., 1979. Introduction à la théorie écologique. Paris, France, Masson, $112 \mathrm{p}$.

24. YONKEU S., 1993. Végétation des pâturages de l'Adamoua (Cameroun) : écologie et potentialités pastorales. Thèse doct., Univ. Rennes I, Rennes, France, 243 p.

25. YOSKO I., 1995. Le système pastoral toubou du Bahr-el-Ghazal (Tchad). Thèse doct., Univ. Sci. Tech. Languedoc, Montpellier, France, $244 \mathrm{p}$.

Reçu le 30.1.97, accepté le 4.8.97

\section{Resumen}

\section{Daget Ph., Poissonet J. Biodiversidad y vegetación pastoril}

Se hace un recordatorio de las normas de calificación de la riqueza específica de la flora de los pastizales, ilustrado de ejemplos mauritanos, burkinos, cameruneses y chadianos. Se examinan las relaciones entre la riqueza de la flora y la productividad, los potenciales del medio y la presión humana y se propone un modelo global de variabilidad.

Palabras clave: Biodiversidad - Flora - Pastoreo - Zona tropical - Medición - Vegetación - Método - Mauritania Burkina Faso - Camerún - Chad. 